Case Reports

\title{
Measles in its Malignant form in a 12-Year-Old Girl Immunosuppressed by HIV at the University Hospital of Brazzaville
}

\author{
${ }^{1,2}$ Ossibi Ibara Bienvenu Rolland, ${ }^{1,2}$ Adoua Doukaga Tatia, \\ ${ }^{1,3}$ Moyen Engoba Mami, ${ }^{2}$ Ekat Martin, ${ }^{2}$ Angonga Pabota Ella, ${ }^{1,3}$ Ekouya Bowassa Gaston, \\ ${ }^{1,3}$ Okoko Annie Rachelle, ${ }^{1,3}$ Mabiala Babela Jean Robert and ${ }^{1,3}$ Mbika Cardorelle Aurore \\ ${ }^{1}$ Faculty of medicine, Marien Ngoabi University, Brazzaville Congo \\ ${ }^{2}$ Infectious Disease Unit, Brazzaville, CHU, Brazzaville Congo \\ ${ }^{3}$ Pediatry Unit, Brazzaville CHU, Brazzaville Congo
}

Article history

Received: 28-12-2019

Revised: 06-02-2020

Accepted: 09-04-2020

Corresponding Author:

Ossibi Ibara Bienvenu Rolland,

Faculty of medicine, Marien

Ngoabi University, Brazzaville

Congo

Email: ossibiibara@gmail.com

\begin{abstract}
Measles is an eruptive and febrile disease that affects unvaccinated or under-vaccinated children, thus undermining the whowanted goal of elimination. Its diagnosis is most often clinical and facilitated by the appearance of a maculopapulus rash associated with at least one element of the naso-oculo-respiratory catarrh in a context of no vaccination. Favourable in the absence of complications, the disease is formidable in its malignant form especially when it occurs in an immune-depressed field especially by HIV. The only cost-effective measure to prevent this disease, in the absence of a contraindication, remains vaccination.
\end{abstract}

Keywords: Measles, HIV, Malignant, CHU, Brazzaville

\section{Introduction}

Measles poses a real public health problem in subSaharan Africa as well as in the Republic of Congo, where weaknesses in the expanded immunization programme are no longer to be demonstrated (OMS, 2008). An immune disease and highly contagious, it most often results in a morbiliform rash in the context of fever associated with a runny nose, eye and sometimes digestive and/or respiratory resulting the classic catarrh naso-oculo digestive or respiratory (WWW.euro.who.int/fr/media-centre). Severe by its complications, it is associated with excess mortality when it occurs in its malignant form on a field previously immunosuppressed by HIV as was the case in a 12-year-old girl admitted to the Infectious Diseases Department of the University Hospital of Brazzaville whose case we report here.

\section{Observation}

This is a 12-year-old female patient, immunosuppressed by TYPE 1 HIV vertically whose mother died. She is on first-line antiretroviral therapy combining AZT-3TC-NVP with the $1 / 1 / 2$ dose twice a day (pediatric form) with CD4 figures at 360 cells $/ \mathrm{mm}^{3}$ and a viral load of 1000 copies. Schooled in sixth grade with a vaccine status with regard to the virus of the genus morbilivirus and tetanus not adjourned, she is admitted to the Department of Infectious Diseases on July 12, 2019 for management of a febrile rash.

The current symptomatology is estimated to date back to six days prior to admission by a fever of $39^{\circ} \mathrm{C}$ associated with diarrhea treated in a local medical practice as a malaria lititry access without established parasitic evidence of injectable quinine, the dose and duration of which have not been clearly elucidated. The patient reportedly received two unwarranted injections of intramuscular gentamicin resulting in the days following widespread pruritus throughout the body. It is in the face of the persistence of the symptomatology made of fever at $39.5^{\circ} \mathrm{C}$ in a context of rhinorrhea and watery and especially the appearance of rashes that the parents finally decide to consult the CHU of Brazzaville hence its admission to the Department of Infectious Diseases.

On arrival, we note a teenage girl in very poor general condition with good skin-mucous coloration, anicteric with persistent skin folds of dehydration and the purpose of undernutrition. The calves are soft and painless. There is no swelling of the lower limbs.

Constants: The temperature is $39^{\circ} \mathrm{C}$, the pulse at 100 beats per minute, the respiratory rate at 28 cycles per
Science

Publications 
minute. $80 \%$ oxygen saturation under the ambient air. The WHO performance status at 3.

The inspection allows to find on the skin plane, a rash made of red maculopapules, one to several millimeters with irregular contours and intervals of healthy skin.
These rashes savers palms of the hands and soles of the feet (Fig. 1). In addition, at the face (Fig. 2), there are skin-mucous hemorrhages such as "black measles". These lesions extend to the gluteal and anal level simulating an associated toxidermy (Fig. 3).

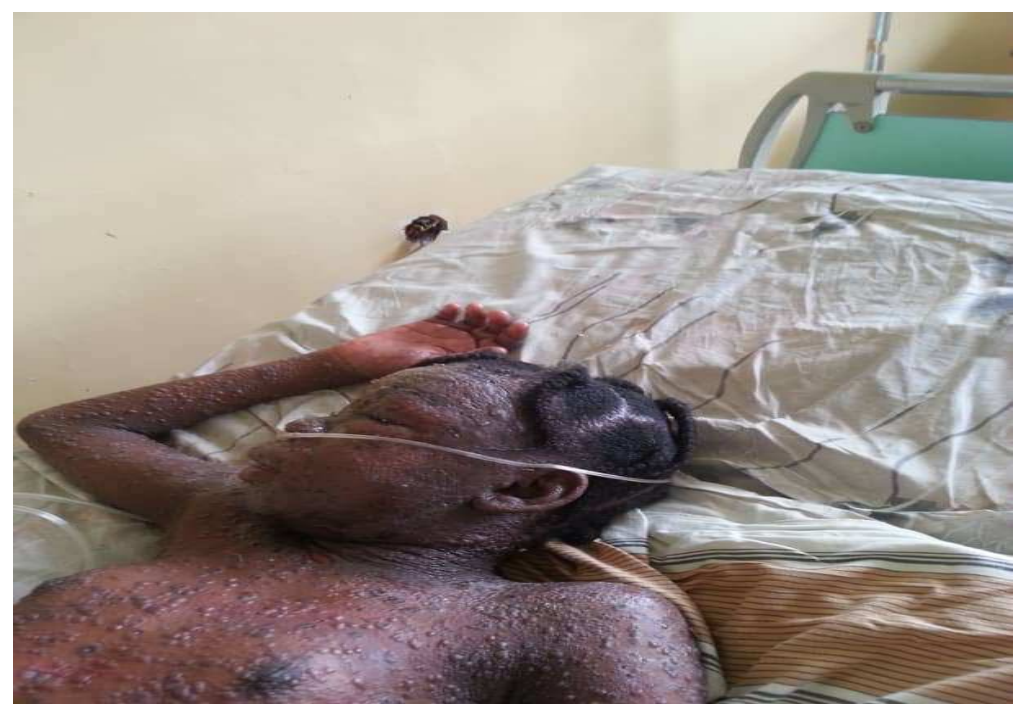

Fig. 1: Maculopapuler eruptions (Photo by Dr Ossibi Ibara)

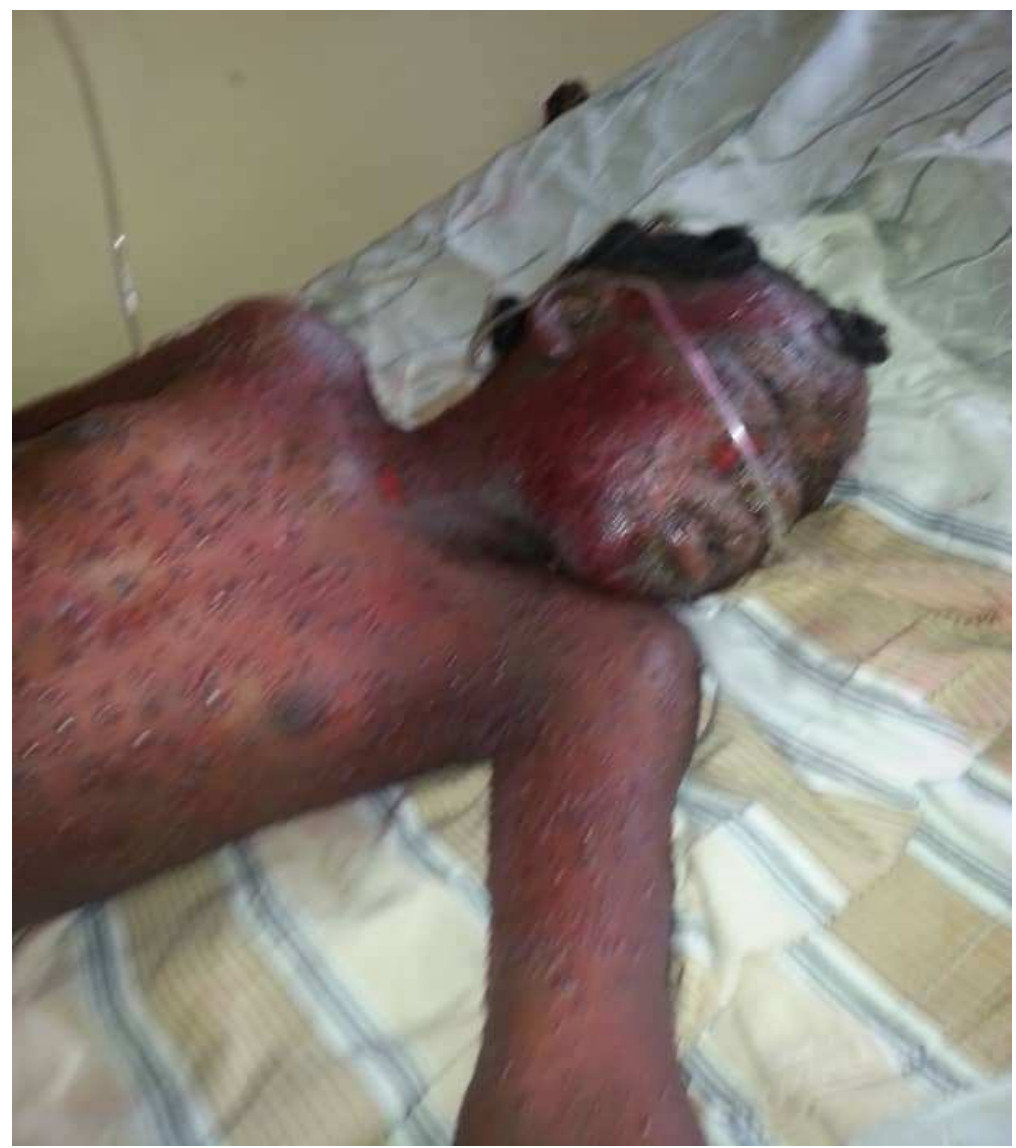

Fig. 2: Eruptions - skin-mucous hemorrhage type "black measles") (Photo Dr. Ossibi Ibara) 


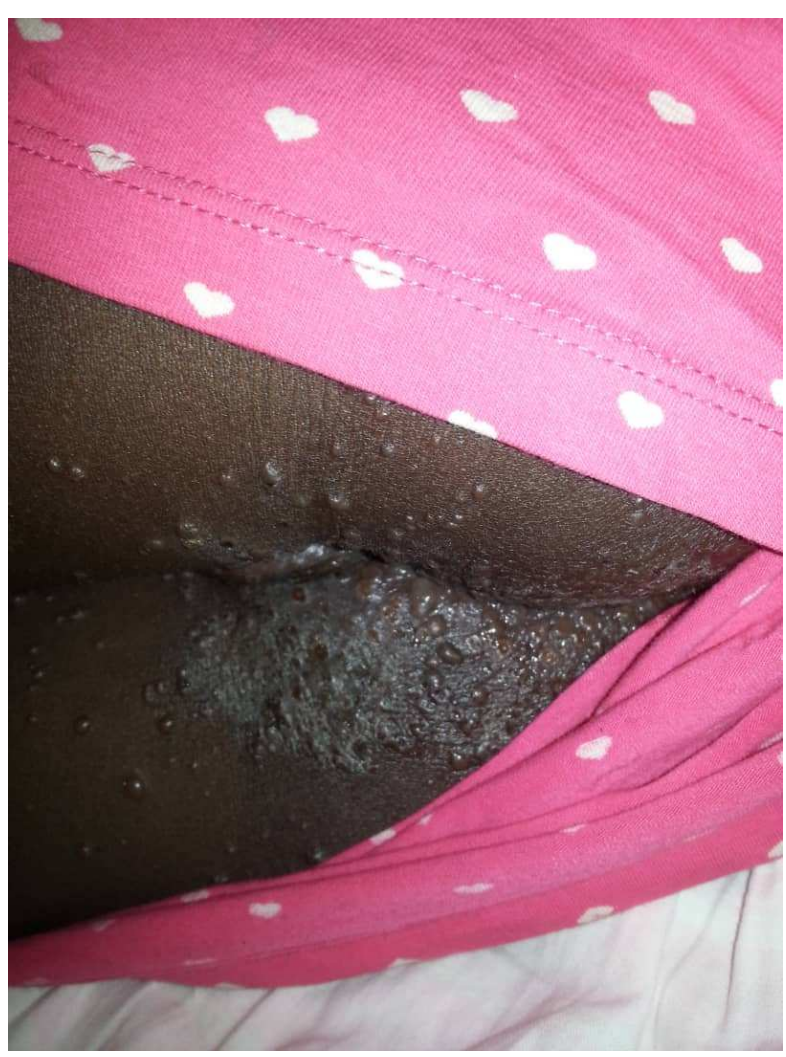

Fig. 3: Anal, maculopapular eruptions (photo by Dr)

Neurologically, the patient is conscious, well oriented in time and space with a Glasgow at 15 . The rest of the physical examination is uncharacteristic

The main course of action was to isolate the patient in an individual ward from the initial consultation in the Infectious Diseases Department. Oxygen therapy at a rate of 6 litres per minute. Disinfection of the nose, and eyes based on an Indocollyra antibiotic eye drops two drops twice a day; vitamin $\mathrm{A}$ at a rate of two doses $24 \mathrm{~h}$ apart according to WHO recommendations (OMS, 2004) and then preventive vaccination and tetanus therapy and ceftriaxone-based antibiotic therapy $2 \mathrm{~g}$ per day to treat possible bacterial overinfections. Under this treatment, the evolution to J19 hospitalization was marked by the disappearance of fever, the flaking of lesions with a tiger-like skin and the patient was exeated at $\mathrm{J} 21$.

\section{Discussion}

Measles continues to pose a real public health problem in the Republic of Congo despite the availability of an effective who-issued vaccine (Ossibi Ibara et al., 2019). She often remains a childhood disease, common in unvaccinated or poorly vaccinated children as was the case in this adolescent. In developing countries, measles is still one of the top 5 causes of death among children under 5 years of age, accounting for more than $90 \%$ of measles deaths (OMS, 2011). The consultation period or even the manifestation of the classic signs sets a long one for our patient. Indeed, HIV infection increases the duration of viremia and especially the lethality of measles, in proportion to the immune deficit and also has an impact on measles vaccination by decreasing the immunological response, making it more imperative to achieve a second dose in the absence of a contraindication (Scott et al., 2007). The clinical signs observed are those most often described in the usual form. The persistence of fever is probably related to bacterial overinfection or HIV infection on its own account (WWW.euro.who.int/fr/media-centre). The absence of measles history and especially prevaccination in an epidemic context in Congo, combined with conventional clinical signs, is sufficient to diagnose measles in the tropiczone. According to WHO recommendations for Integrated Management of Childhood Diseases (IMCI) in developing countries, measles diagnosis can be made in the presence of a generalized maculo-papuleux febrile exanthema associated with one of the signs: cough, rhinorrhea, conjunctivitis (OMS, 2011). Measles treatment is only symptomatic as was the case in the patient and notified by WHO (OMS, 2004). The patient's length of hospital stay span, probably related to the subjacent terrain on which the disease occurs. It averages 6.84-1 days in the same ward in a population of children aged an average of 2.5-2.6 living in Brazzaville (Ossibi Ibara et al., 2019). The evolution of measles outside of complications is classically favourable. In the same ward, $89.5 \%$ of children with measles had a favourable.

\section{Conclusion}

The epidemiology and clinic of measles remain classic in Congo where the disease continues to pose a real problem of Public Health. HIV immunodepression increases the duration of viremia and can be a fatal cause of the disease in proportion to immune deficiency. It is therefore important to vaccinate children in the absence of immunodepression in order to reduce the morbimortality associated with this infection

\section{Author's Contributions}

Ossibi Ibara Bienvenu Rolland: To initiated the work, write the article. Coordinated the data analysis and contributed to the writing of the manuscript.

Adoua Doukaga Tatia: To contributed to the collection of data.

Moyen Engoba Mami and Ekat Martin: Coordinated the mouse work.

Angonga Pabota Ella and Ekouya Bowassa Gaston: Contributed to the writing of the manuscript. 
Okoko Annie Rachelle, Mabiala Babela Jean Robert and Mbika Cardorelle Aurore: Cordinated the data analysis and contributed of the writing of the manuscript.

\section{Conflict of Interest}

The authors repair no conflict of interest in relation to this study.

\section{Ethical Rule}

We obtained the free and informed consent of the patient and her biological father to proceed with the publication of this study and the images associated with it.

\section{References}

OMS, 2004. Le treatment de la rougeole. WHO/EPI/TRAM/97.02

OMS, 2008. Progrès accomplis dans la lutte contre la rougeole et la réduction de la mortalité rougeoleuse au niveau mondial-2000-2007. 83: 441-447.
OMS, 2011. Flambées de rougeole et progrès accomplis en vue d'atteindre les objectifs de pré élimination de rougeole: Région africaine de l'OMS, 2009-2010. Wkly Epide Record, 86: 129-140.

Ossibi Ibara, B.R., C.A. Attinsounon, P.W. Atipo-Tsiba, T. Adoua Doukaga and L.C. Ollandzobo et al., 2019. Measles: Épidemiological characteristics and associated factors of patients admitted to the Infectious Diseases unit at the Brazzaville University Hosptital. Am. J. Infect. Dis. Microbiol., 7: 13-17.

Scott, S., W.J. Moss, S. Cousens, 2007. The influence of HIV-1 exposure and infection on levels of passively acquired antobodies to measles virus in Zambian infants. Clin. Infect. Dis., 45: 1417-1424.

WWW.euro.who.int/fr/media-centre: sections/pressreleases: 2018/measles-cases-hit-record-high-in-theEuropean-region 